\title{
Pharmacokinetic thoughts on the repurposing of oral ivermectin for treatment of COVID-19
}

\author{
Xiao Zhu ${ }^{1}$, Stephen Duffull ${ }^{1}$, Andrew Steer ${ }^{2}$, and Amanda Gwee ${ }^{3}$ \\ ${ }^{1}$ University of Otago \\ ${ }^{2}$ Murdoch Childrens Research Institute \\ ${ }^{3}$ The Royal Children's Hospital Melbourne
}

April 28, 2020

\begin{abstract}
None

A recent commentary published in BJCP used lopinavir/ritonavir as an example to highlight the importance of the clinical pharmacology principles in the repurposing of old drugs for therapeutic use against Coronavirus disease 19 (COVID-19). ${ }^{1}$ Here, we provide another example to support this point.

A recent study found that ivermectin, an FDA-approved anti-parasitic drug, has inhibitory effects on the severe acute respiratory syndrome coronavirus 2 (SARS-CoV-2). ${ }^{2}$ Ivermectin has broad anti-viral activity through inhibition of viral proteins including importin $\alpha / \beta 1$ heterodimer and integrase protein. ${ }^{3}$ In the in vitro study reported by Caly and colleagues, the addition of ivermectin at a concentration of 5 micromolar $(\mu \mathrm{M})$ (twice the reported IC50) to Vero-hSLAM cells 2 hours post infection of with SARS-CoV-2 resulted in a reduction in the viral RNA load by $99.98 \%$ at 48 hours. $^{2}$

Large trials of mass drug administration of ivermectin in adults and children have shown that ivermectin is well tolerated. ${ }^{4}$ Even at doses that are 10 times greater than the highest FDA-approved dose of $200 \mu \mathrm{g} / \mathrm{kg}$, central nervous system toxicity has not been reported. ${ }^{5}$ However, following the oral administration of supratherapeutic doses of ivermectin (i.e. $120 \mathrm{mg}$ ) the maximum plasma concentration achieved was $0.28 \pm 0.18$ (standard deviation) $\mu \mathrm{M}$, a value 18 times lower than the reported $5 \mu \mathrm{M}$ ivermectin concentration used by Caly et al in their SARS-CoV-2 experiment. ${ }^{5}$ To date, the clinical effects of ivermectin at a concentration of $5 \mu \mathrm{M}$ range are unknown, but likely to be toxic. Furthermore, ivermectin is only commercially available as a $3 \mathrm{mg}$ oral tablet. ${ }^{6}$ These factors hinder our ability to immediately repurpose ivermectin in its current form for the treatment of COVID-19.

While the findings by Caly and colleagues provide some promise, viral suppression was not seen at concentrations observed with standard doses in humans. Further preclinical in vivo studies should evaluate the pharmacokinetics and pharmacodynamics to determine the kill pattern of ivermectin. A potential alternate solution may be to develop an inhaled formulation of ivermectin to efficiently deliver a high local concentration in the lung, whilst minimising systemic toxicity. As therapeutic agents to tackle the COVID19 pandemic are urgently sought, careful consideration of the pharmacokinetics of these drugs should be considered to guide in vitro testing.
\end{abstract}

\section{Conflict of interest}

None

\section{References:}


1. Smith PF, Dodds M, Bentley D, et al. Dosing will be a key success factor in repurposing antivirals for COVID-19. Br J Clin Pharmacol . 2020 Apr 17. doi: 10.1111/bcp.14314

2. Caly L, Druce JD, Catton MG, et al. The FDA approved Drug Ivermectin inhibits the replication of SARS-CoV-2 in vitro. Antivir Res2020 https://doi.org/10.1016/j.antiviral.2020.104787.

3. Wagstaff KM, Rawlinson SM, Hearps AC, et al. An AlphaScreen@-based assay for high-throughput screening for specific inhibitors of nuclear import. J Biomol Screen 2011; 16(2): 192-200

4. Weil GJ, Bogus J, Christian M, et al. The safety of double- and triple-drug community mass drug administration for lymphatic filariasis: A multicenter, open-label, cluster-randomized study. PLoS Med 2019;16(6):e1002839.

5. Guzzo CA, Furtek CI, Porras AG, et al. Safety, tolerability, and pharmacokinetics of escalating high doses of ivermectin in healthy adult subjects. J Clin Pharmacol 2002; 42(10): 1122-1133.

6. U.S. Package Circular: STROMECTOL@ (ivermectin). Whitehouse Station, NJ: Merck \& Co., Inc., 1998. 